\title{
Green Cloud -The Safe Prospect in Computing
}

\author{
D. Chandrika \\ Asst. Professor, Dept. of CSE Vignan's Institute of Engg. For Women \\ Visakhapatnam, Andhra Pradesh, India,
}

\section{Kamal Kumari}

Asst. Professor, Dept. of CSE Vignan's Institute of Engg. For Women Visakhapatnam, Andhra Pradesh, India,

Nandita Bhanja Chaudhuri

Asst. Professor, Dept. of IT Vignan's Institute of Engg. For Women

Visakhapatnam, Andhra Pradesh, India,

Sadi Ram Prasad Reddy Assoc. Professor Dept. of CSE

Vignan's Institute of Engg. For Women Visakhapatnam, Andhra Pradesh, India,

\begin{abstract}
The growing demand of Cloud infrastructure has drastically increased the energy consumption of data centers, that has become a critical issue, leading to high carbon emissions which is harmful for environment. Hence, energy-efficient solutions are required to minimize the impact of Cloud computing on the environment. One solution is implementation of Global Green Mantra. The Global Green Mantra states Reduce, Reuse and Recycle Green cloud refers to the potential environmental benefits the information technology (IT) services delivered over the Internet to the society. The term Green Cloud combines the words green -meaning environmentally friendly -- and cloud, the traditional symbol for the Internet and the shortened name for a kind of service delivery model known as cloud computing. This paper proposes an energy-alert layer in software architecture that is responsible for reviewing micro-metrics and macro-metrics of energy consumption in data centers and then makes services to migrate to hosts consuming energy more efficiently.
\end{abstract}

\section{INTRODUCTION}

Cloud computing provides computing power and resources as a service to users across the globe. In recent times cloud computing [2] has fascinated significant attention in society. Cloud computing is a model for enabling convenient, ondemand network access to a shared pool of configurable computing resources that can be rapidly provisioned and released with minimal management effort or service provider interaction. By this means, customers will be able to access applications and data from a "cloud" anywhere all over the world .The increasing availability of high-speed Internet and corporate IP connections is enabling the delivery of new network-based services [4]. While Internet-based mail services have been operating for many years, service offerings have recently expanded to include network-based storage and network-based computing. These new services are being offered both to corporate and individual end users [5], [6]. Services of this type have been generically called "cloud computing" services [5]-[13].

Cloud computing can be considered as a hierarchy of concepts, which comprises of several models. The first model is the Service Model [14] which further includes three models namely -software as a service, platform as a service and infrastructure as a service. Second is the Deployment model [11] which further comprises of public cloud, private cloud, community cloud and hybrid cloud.

Global warming has been a big concern of late, with high power consumption and $\mathrm{CO}_{2}$ emission acting as a catalyst to increase the same. The world has become highly protective about the environment with inputs from contributors such as Greenpeace, Environmental Protection Agency (EPA) of the United States and the Climate Savers Computing Initiative to name a few. With the continuously increasing popularity and usage of cloud computing and the increasing awareness of the people across the globe towards the use of eco-friendly resources has forced the researchers to devise concepts towards an eco-friendly energy efficient flavor of cloud computing called green cloud computing. According to the previous works green cloud computing facilitates the reduction of power consumption and $\mathrm{CO}_{2}$ emission along with the reutilization of energy in an efficient way

Recently, high performance has been the sole concern in data center deployments and this demand has been fulfilled without paying much attention to energy consumption. The average data center consumes as much energy as 25,000 
households. As energy costs are increasing while availability dwindles, there is a need to shift focus from optimizing data center resource management for pure performance to optimizing for energy efficiency while maintaining high service level performance. According to certain reports, the total estimated energy bill for data centers in 2010 is $\$ 11.5$ billion and energy costs in a typical data center double every five years.

Data centers are not only expensive to maintain, but also unfriendly to the environment. Data centers now drive more in carbon emissions than both Argentina and the Netherlands. High energy costs and huge carbon footprints are incurred due to massive amounts of electricity needed to power and cool numerous servers hosted in these data centers. Cloud service providers need to adopt measures to ensure that their profit margin is not dramatically reduced due to high energy costs. For instance, Google, Microsoft, and Yahoo are building large data centers in barren desert land surrounding the Columbia River, USA to exploit cheap and reliable hydroelectric power. Lowering the energy usage of data centers is a challenging and complex issue because computing applications and data are growing so quickly that increasingly larger servers and disks are needed to process them fast enough within the required time period. Green Cloud computing is envisioned to achieve not only efficient processing and utilization of computing infrastructure, but also minimize energy consumption. This is essential for ensuring that the future growth of Cloud computing is sustainable. Otherwise, Cloud computing with increasingly pervasive front-end client devices interacting with back-end data centers will cause an enormous escalation of energy usage.

To address this problem, data center resources need to be managed in an energy-efficient manner to drive Green Cloud computing. In particular, Cloud resources need to be allocated not only to satisfy QoS requirements specified by users via Service Level Agreements (SLA), but also to reduce energy usage.

\section{Green Cloud Architecture}

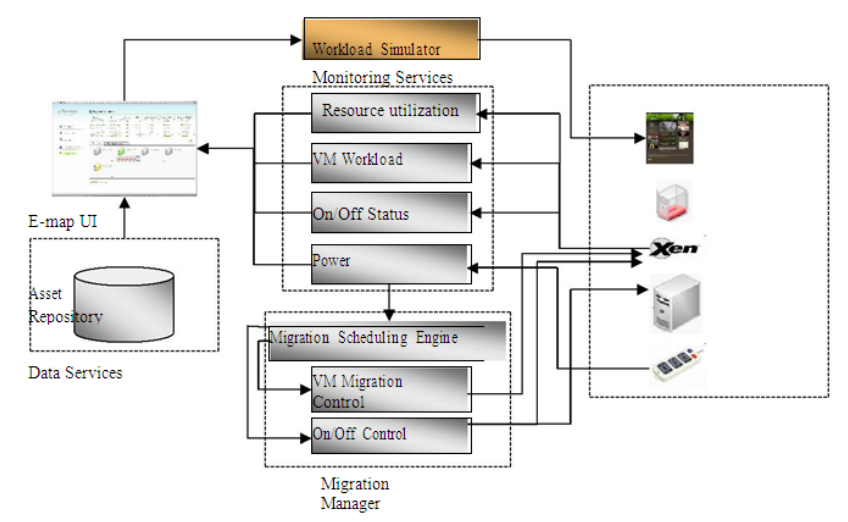

Figure 1. GreenCloud Architecture

As discussed above, cloud computing platform as the next generation IT infrastructure enables enterprises to consolidate computing resources, reduce management complexity and speed the response to business dynamics. Improving the resource utilization and reduce power consumption are key challenges to the success of operating a cloud computing environment. To address such challenges, we design the GreenCloud architecture and the corresponding GreenCloud exploratory system. The exploratory system monitors a variety of system factors and performance measures including application workload, resource utilization and power consumption, hence the system is able to dynamically adapt workload and resource utilization through VM live migration. Therefore, the GreenCloud architecture reduces unnecessary power consumption in a cloud computing environment. Figure 2 demonstrates the GreenCloud architecture and shows the functions of compone nts and their relations in the architecture.

Monitoring Service monitors and collects comprehensive factors such as application workload, resource utilization and power consumption, etc. The Monitoring Service is built on top of IBM Tivoli framework and Xen, where the IBM Tivoli framework is a CORBA-based system management platform managing a large number of remote locations and devices; Xen is a virtual machine monitor (VMM). The Monitoring Service serves as the global information provider and provides on-demand reports by performing the aggregation and pruning the historical raw monitoring data to support to intelligent actions taken by Migration Manager.

Migration Manager triggers live migration and makes decision on the placement of virtual machines on physical servers based on knowledge or information provided by the Monitoring Service. The migration scheduling engine searches the optimal placement by a heuristic algorithm, and sends instructions to execute the migration and turn on or off a server. A heuristic algorithm to search an optimal VM placement and the implementation details of Migration Manager will be discussed in Section IV. The output of the algorithm is an action list in terms of migrate actions (e.g.

Migrate VM1 from PM2 to PM4) and local adjustment actions (e.g. Set VM2 CPU to $1500 \mathrm{MHz}$ [ [40].

Managed Environment includes virtual machines, physical machines, resources, devices,remote commands on VMs, and applications with adaptive workload, etc.

E-Map is a web-based service with Flash front-end. It provides a user interface (UI) to show the real -time view of present and past system on/off status, resource consumption, workload status, temperature and energy consumption in the system at multiple scales, from high-level overview down to individual IT devices (e.g. servers and storage devices) and other equipment (e.g. water- or air-cooling devices). E-map is connected to the Workload Simulator, which predicts the consequences after a given actions adopted by the Migration Monitor through simulation in real environment.

Workload Simulator accepts user instructions to adapt workload, e.g. CPU utilization, on servers, and enables the control of Migration Manager under various workloads. Then, E-Map collects the corresponding real -time measurements, and demonstrates the performance of the system to users. Therefore, users and system designers will verify the effectiveness of a certain algorithm or adjust parameters of the algorithm to achieve better performance. 
Asset Repository is a database to store the static server information, such as IP address, type, CPU configuration, memory setting, and topology of the servers.

The GreenCloud IDC management frame work is running and accessible to IBM internal staffs and customers. They can view up-to-date status of resources, configure their applications, allocate resources, and experience the live management system.

\section{APPROACHES TO GREEN COMPUTING}

These paths intertwine and are all vital to help the environment

- Green Use - Reducing the Energy Consumption of computers and use them in a environmental manner.

- Green Disposal - refurbish and reuse old computers as well as recycling them.

- Green Design - Designing Energy efficient components, computers, servers, and other electronic equipment.

- Green Manufacturing - manufacturing equipment while leaving a minimal footprint.

Energy costs of IT and data center operations are significant, whether for internal corporate IT operations or as part of IT outsourcing, Power consumption, Cooling, "Inefficient" equipment operations, e.g., data servers "spinning" when no active operations are being performed. In "old days" energy costs were assumed to be free. In current environment (pun intended), equipment costs have been reduced, putting focus on energy costs.

\subsection{Virtualization:}

Initiatives in this area include server virtualization and consolidation, storage consolidation and desktop virtualization. These projects typically improve cost and energy efficiency through optimized use of existing and new computing and storage capacity, electricity, cooling, ventilation and real estate. Moving desktops to a virtual environment and employing thin-client machines reduces energy consumption and environmental impact of user infrastructure. As one senior partner at a 100-employee services firm reports, "[Thin clients have] no CPU, no RAM, no moving parts, and connect to the virtual desktop environment. Our typical computer used up to a 250-watt power supply; our thin client uses a 4.8-watt power supply, so the reduction in electricity usage is 97,98 percent, with all the functionality. "Energy savings result, as does cost avoidance, thanks to extended refresh cycles provided by thin client equipment. Mid-size businesses face a preponderance of issues when it comes to the server room. In this study, businesses cite the following reasons for undertaking server room upgrades and the construction of new server rooms:

- Decrease cost and increase effectiveness of cooling and ventilation systems. Many existing HVAC systems cannot keep up with smaller, more powerful servers that throw off more heat than older, low-density equipment. Most server rooms were not designed to keep pace with the modern complement of fully virtualized servers and consolidated storage.
- Increase server and computing capacity. Server room spaces are simply maxed out; they are either too small to house needed servers, or inadequately equipped to deal with a high rate of virtualization on fewer devices that run hotter.

- Questionable reliability of aging server room infrastructure; the server room design of yesterday no longer supports business needs of today, in terms of uptime and availability.

- Mounting maintenance and management costs for older facilities, which may not affordably handle growth of computing and storage.

- The need to decrease real estate costs, through server room infrastructure that supports denser, smaller footprints of new servers and storage.

Computer virtualization is the process of running two or more logical computer systems on one set of physical hardware. The concept originated with the IBM mainframe operating systems of the $1960 \mathrm{~s}$, but was commercialized for $\mathrm{x} 86$ compatible computers only in the 1990 s. With virtualization, a system administrator could combine several physical systems into virtual machines on one single, powerful system, thereby unplugging the original hardware and reducing power and cooling consumption. Several commercial companies and open-source projects now offer software packages to enable a transition to virtual computing. Intel Corporation and AMD have also built proprietary virtualization enhancements to the $\mathrm{x} 86$ instruction set into each of their CPU product lines, in order to facilitate virtualized computing. Server Virtualisation increases network utilization and reduces network equipment needs by allowing multiple virtual servers to share one or more network adapters within the confines of a single physical server.

\begin{tabular}{|l|c|c|}
\hline Company & Servers Before & Servers After \\
\hline Tourism Aust & 70 & 35 \\
\hline Stirling Council WA & 54 & 7 \\
\hline Brisbane Council & 85 & 20 \\
\hline Orica & 40 & 10 \\
\hline Townsville Council (old) & 100 & 53 \\
\hline
\end{tabular}

\section{Fig 3.Companys implementing Green computing Techniques}

\subsection{PC POWER MANAGEMENT:}

Many look to managing end-user device power consumption as an easy, effective way to reduce energy costs. These power management initiatives include the following:

- Using software that centrally manages energy settings of PCs and monitors.

- Enforcing standardized power settings on all PCs before distributing to end users.

- Procuring energy-efficient equipment, such as Energy Star certified devices.

- Power management for computer systems are desired for many reasons, particularly:

- $\quad$ Prolong battery life for portable and embedded systems. 
- Reduce cooling requirements.

- Reduce noise.

- Reduce operating costs for energy and cooling.

- Lower power consumption also means lower heat dissipation, which increases system stability, and less energy use, which saves money and reduces the impact on the environment.

- The Advanced Configuration and Power Interface (ACPI), an open industry standard, allows an operating system to directly control the power saving aspects of its underlying hardware. This allows a system to automatically turn off components such as monitors and hard drives after set periods of inactivity. In addition, a system may hibernate, where most components (including the CPU and the system RAM) are turned off. ACPI is a successor to an earlier Intel-Microsoft standard called Advanced Power Management, which allows a computer's BIOS to control power management functions.

- Some programs allow the user to manually adjust the voltages supplied to the CPU, which reduces both the amount of heat produced and electricity consumed.

This process is called undervolting.

Some CPUs can automatically undervolt the processor depending on the workload; this technology is called "SpeedStep" on Intel processors, "PowerNow!"/"Cool'n'Quiet" on AMD chips, LongHaul on VIA CPUs, and LongRun with Transmeta processors. The power management for microprocessors can be done over the whole processor, or in specific areas. With dynamic voltage scaling and dynamic frequency scaling, the CPU core voltage, clock rate, or both, can be altered to decrease power consumption at the price of slower performance. This is sometimes done in real time to optimize the powerperformance tradeoff.

Examples:

- Intel SpeedStep

- AMD Cool'n'Quiet

- AMD PowerNow!

- VIA LongHaul (PowerSaver)

- Transmeta LongRun and LongRun2

Newer Intel Core processors support ultra-fine power control over the function units within the processors.

\subsection{POWER SUPPLY:}

Power supplies in most computers (PSUs for short) aren't designed for energy efficiency. In fact, most computers drain more power than they need during normal operation, leading to higher electrical bills and a more dire environmental impact. The 80 Plus program is a voluntary certification system for power-supply manufacturers. The term "80 Plus" is a little complicated, so bear with me for a moment. If a PSU meets the certification, it will use only the power it needs at a given load: In other words, it won't use more power than it needs. For example, if your PC requires only 20 percent of the total power of a 500-watt PSU, the system will consume no more than 100 watts. Only when the PC requires full power will the PSU run at the full wattage load. An 80 Plus power supply can save about 85 kilowatt hours per PC, per year. In many ways, it's the heart of a green PC, since it manages the power for all the other components. It also has the most dramatic effect on your energy bill. Of course, all 80 Plus power supplies are also lead-free and RoHS compliant.

Desktop computer power supplies (PSUs) are generally 70 $75 \%$ efficient, dissipating the remaining energy as heat. An industry initiative called 80 PLUS certifies PSUs that are at least $80 \%$ efficient; typically these models are drop-in replacements for older, less efficient PSUs of the same form factor. As of July 20, 2007, all new Energy Star 4.0-certified desktop PSUs must be at least $80 \%$ efficient.

Various initiatives are underway to improve the efficiency of computer power supplies. Climate savers computing initiative promotes energy saving and reduction of greenhouse gas emissions by encouraging development and use of more efficient power supplies.

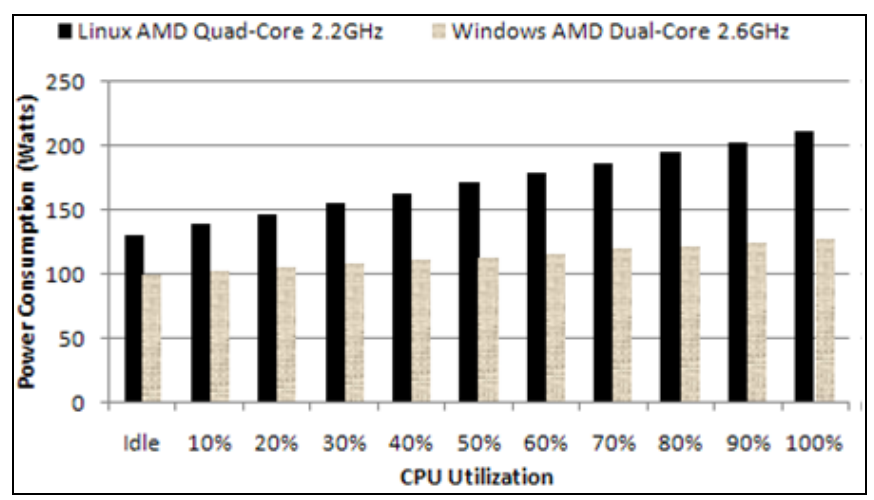

Fig 1: Power consumption under different workloads.

\subsection{STORAGE:}

There are three routes available, all of which vary in cost, performance, and capacity. The most conventional route is the 3.5" desktop hard drive. Recently, major drive manufacturers have begun to focus on reduced power consumption, resulting in such features as the reduced RPM low-power idle mode with fixed rotation speed for reduced power consumption. The advantages of this route are the highest possible capacity, the best performance (out of the highest-end solid-state drives). The second option, which also lends itself to affordability, is to use a 2.5" laptop hard drive. These consume less power than larger disks as a result of their smaller platters, smaller motors, and firmware that is already optimized for power consumption versus most 3.5" harddisks. With capacities up to $320 \mathrm{~GB}$, reasonable capacity is well within reach, although the price is substantially higher than an equivalent 3.5" disk. With a green system aimed at light use, a 120GB or $160 \mathrm{~GB}$ laptop drive is a very affordable, lower-power alternative to a 3.5" disk. The lowest-power option is to use a solid state hard drive (SSD), which typically draw less than one-third the power of a 2.5 " disk. The latest, highest-performance SSDs are very fast but extremely expensive, and currently top out at only 64GB. That's adequate for light use, but wholly inadequate for gamer, video editing, and other heavy uses. More affordable SSDs are available in larger capacities, but are not cheap and typically have slow write performance, 
which limits their practical utility. Smaller form factor (e.g. 2.5 inch) hard disk drives often consume less power than physically larger drives. Unlike hard disk drives, solid-state drives store data in flash memory or DRAM. With no moving parts, power consumption may be reduced somewhat for low capacity flash based devices. Even at modest sizes, DRAM based SSDs may use more power than hard disks, (e.g., 4GB i-RAM uses more power and space than laptop drives). Flash based drives are generally slower for writing than hard disks.

\subsection{VIDEO CARD:}

A fast GPU may be the largest power consumer in a computer. Energy efficient display options include:

- No video card - use a shared terminal, shared thin client, or desktop sharing software if display required.

- Use motherboard video output - typically low 3D performance and low power.

- Reuse an older video card that uses little power; many do not require heatsinks or fans.

Select a GPU based on average wattage or performance per watt. The easiest way to conserve power is to go with integrated video. This is the lowest performance option, but for office users, casual browsing, and pure 2D use, it's more than adequate and well worth saving the $10 \mathrm{~W}, 20 \mathrm{~W}$, or even $35 \mathrm{~W}$ from a discrete video card. Motherboards spitting out integrated video via DVI or HDMI aren't that hard to find, so power-users with their massive LCDs don't have to suffer.

\subsection{DISPLAYS:}

LCD monitors typically use a cold-cathode fluorescent bulb to provide light for the display. Some newer displays use an array of light-emitting diodes (LEDs) in place of the fluorescent bulb, which reduces the amount of electricity used by the display. LCD monitors uses three times less when active, and ten times less energy when in sleep mode. LCDs are up to $66 \%$ more energy efficient than CRTs, LCDs are also upwards of $80 \%$ smaller in size and weight, leading to fuel savings in shipping. LCDs produce less heat, meaning you'll need less AC to keep cool. LCD screens are also easier on the eyes. Their lower intensity and steady light pattern result in less fatigue versus CRTs. A newer LCD draws 40$60 \mathrm{~W}$ maximum in a modest 19", 20", or 22" size. That number grows close to $85 \mathrm{~W}$ or $100 \mathrm{~W}$ maximum for a $24 "$ unit. Drop them down to standby or turn them off entirely when not using them to minimize power consumption. By comparison, a 21 " CRT typically uses more than $120 \mathrm{~W}$, more than double the power of a typical 22" LCD.

\subsection{IT EQUIPMENT RECYCLING:}

After you've finished with your IT products, what happens when they're no longer needed? In nature, organic materials rot down and feed future growth, so why not dismantle products at the end of their lives and use the elements as raw materials for future products? Several reputable computer manufacturers use metal and easily separated plastics in order to maximise raw material reuse. It's important that the environmental costs of recovery don't exceed the benefits expected. And that, of course, loops back to design in the first place. The priorities for all material things are reduce, reuse and recycle - in that order of importance. If you can extend the working life of your IT products, you reduce the environmental consequences of mining, manufacture, packaging, shipping and disposal. Can you upgrade something rather than finish using it? If you have to replace it, can someone else inside your organisation use it? If not, charities and refurbishing organisations may be able to extend the product's life. And, waiting at the end of the line, many organisations, including some manufacturers themselves, are willing to take equipment back and recycle the components into new products. Out of all initiatives in this study, the success of IT equipment recycling relies not on a business case with cost savings, but on a combination of environmental responsibility and regulatory pressures. The single most important factor in adopting recycling initiatives is to decrease waste sent to landfills.

Recycling computing equipment can keep harmful materials such as lead, mercury, and hexavalent chromium out of landfills. Obsolete computers are a valuable source for secondary raw materials, if treated properly, however if not treated properly they are a major source of toxins and carcinogens. Rapid technology change, low initial cost and even planned obsolescence have resulted in a fast growing problem around the globe. Technical solutions are available but in most cases a legal framework, a collection system, logistics and other services need to be implemented before a technical solution can be applied. Electronic devices, including audio-visual components (televisions, VCRs, stereo equipment), mobile phones and other handheld devices, and computer components, contain valuable elements and substances suitable for reclamation, including lead, copper, and gold. They also contain a plethora of toxic substances, such as dioxins, PCBs, cadmium, chromium, radioactive isotopes, and mercury. Additionally, the processing required to reclaim the precious substances (including incineration and acid treatments) release, generate and synthesize further toxic byproducts Most major computer manufacturers offer some form of recycling, often as a free replacement service when purchasing a new PC. At the user's request they may mail in their old computer, or arrange for pickup from the manufacturer.

Individuals looking for environmentally-friendly ways in which to dispose of electronics can find corporate electronic take-back and recycling programs across the country. Open to the public (in most cases), corporations nationwide have begun to offer low-cost to nocost recycling, and have opened centers nationally and in some cases internationally. Such programs frequently offer services to take-back and recycle electronics including mobile phones, laptop and desktop computers, digital cameras, and home and auto electronics. Companies offer what are called "take-back" programs that provide monetary incentives for recyclable and/or working technologies. While there are several health hazards when it comes to dealing with computer recycling some of the substances you should be aware of: 
- $\quad$ Lead common in CRTs, older solder, some batteries and to some formulations of PVC. Can be harmful if not disposed of properly.

- Mercury in fluorescent tubes. With new technologies arising the elimination of mercury in many new model computers is taking place.

- Cadmium in some rechargeable batteries. It can be hazardous to your skin if exposed for too long. Although many people are exposed to it everyday it just depends on the amount of exposure.

- Liquid crystals are another health hazard that should be taken into consideration although they do not have the nearly the same effects as the other chemicals.

\section{HOW TO IMPLEMENT?}

\section{Greening Your Organization:}

The whole idea of replacing physical movement with electronic communications like videoconferencing reduces environmental impacts, not to mention associated costs. This also applies to how you manage your business processes. Consider distributing information electronically rather than printing it first and then distributing it. This 'print on demand' approach saves transport and unnecessary copies, not to mention saving money! Companies with transport and logistics operations can reduce emissions by using software applications to optimise routes and eliminate wasted journeys. Solutions can range from simple sat-nav devices to more complex transportation management systems which coordinate multiple vehicles and routes, saving both time and fuel, and providing more predictable customer service too. Power management softwares help the computers to sleep or hibernate when no in use. Reversible computing (which also includes quantum computing) promises to reduce power consumption by a factor of several thousand, but such systems are still very much in the laboratories. Reversible computing includes any computational process that is (at least to some close approximation) reversible, i.e., time-invertible, meaning that a time-reversed version of the process could exist within the same general dynamical framework as the original process. Reversible computing's efficient use of heat could make it possible to come up with 3-D chip designs, Bennett said. This would push all of the circuitry closer together and ultimately increase performance.

\section{Nearing green nirvana}

- Audit your data centre and remove unused equipment and software

- Virtualise applications, storage and servers in the data centre wherever appropriate

- Consider consolidating data centres

- Introduce videoconferencing or telepresence facilities.

- Power-sucking displays can be replaced with green light displays made of OLEDs, or organic light-emitting diodes.

- Use of toxic materials like lead can be replaced by silver and copper.

- Making recycling of computers (which is expensive and time consuming at present) more effective by recycling computer parts separately with an option of reuse or resale.

- Buy and use a low power desktop or a laptop computer (40-90 watts) rather a higher power desktop (e.g. 300 watts).

- Thin clients can use only 4 to 8 watts of power at the desktop as the processing is done by a server.

- For desktops, buy a low power central processing unit (CPU). This reduces both power consumption and cooling requirements.

- Buy hardware from manufacturers that have a hardware recycling scheme, and recycle your old computer equipment rather than sending it to landfill.

- Turn your computer and monitor off when you are not using it.

- Enable hibernation using the power management settings. Standby does not save as much power.

- Replace your CRT screen with an LCD screen.

- Keep your PC or laptop for at least 5 years. If you're leasing, shift to a 5 year period. This reduces energy consumption by $40 \%$, compared to replacing PCs every 3 years which is current corporate practice.

- Avoid an unnecessary operating system version upgrade which requires a hardware upgrade.

- Use Linux (such as Ubuntu), which requires less resources than many other operating systems on an older computer as a spare or a file server.

- Use server virtualization to aggregate multiple underutilized servers onto more energy efficient server infrastructure.

\section{FUTURE OF GREEN COMPUTING}

As $21^{\text {st }}$ century belongs to computers, gizmos and electronic items, energy issues will get a serious ring in the coming days, as the public debate on carbon emissions, global warming and climate change gets hotter. If we think computers are nonpolluting and consume very little energy we need to think again. It is estimated that out of $\$ 250$ billion per year spent on powering computers worldwide only about $15 \%$ of that power is spent computing- the rest is wasted idling. Thus, energy saved on computer hardware and computing will equate tonnes of carbon emissions saved per year. Taking into consideration the popular use of information technology industry, it has to lead a revolution of sorts by turning green in a manner no industry has ever done before. Opportunities lie in green technology like never before in history and organizations are seeing it as a way to create new profit centers while trying to help the environmental cause. The plan towards green IT should include new electronic products and services with optimum efficiency and all possible options towards energy savings. Faster processors historically use more power. Inefficient CPU's are a double hit because they both use too much power themselves and their waste heat increases air conditioning needs, especially in server farms-between the computers and the HVAC. The waste heat also causes reliability problems, as CPU's crash much more often at highe temperatures. Many people have been working for years to lice this inefficiency out of computers. Similarly, 
power supplies are notoriously bad, generally as little as $7 \%$ efficient. And since everything in a computer runs off the power supply, nothing can be efficient without a good power supply. Recent inventions of power supply are helping fix this by running at $80 \%$ efficiency or better.

\section{INDUSTRIAL IMPLEMENTATIONS OF GREEN COMPUTING \\ 6.1 Blackle:}

Blackle is a search-engine site powered by Google Search. Blackle came into being based on the concept that when a computer screen is white, presenting an empty word page or the Google home page, your computer consumes $74 \mathrm{~W}$. When the screen is black it consumes only $59 \mathrm{~W}$. Based on this theory if everyone switched from Google to Blackle, mother earth would save $750 \mathrm{MW}$ each year. This was a really good implementation of Green Computing. The principle behind Blackle is based on the fact that the display of different colors consumes different amounts of energy on computer monitors.

\subsection{Fit-PC: a tiny PC that draws only $5 w$ :}

Fit-PC is the size of a paperback and absolutely silent, yet fit enough to run Windows XP or Linux. fit-PC is designed to fit where a standard $\mathrm{PC}$ is too bulky, noisy and power hungry. If you ever wished for a PC to be compact, quiet and green then fit- PC is the perfect fit for you. Fit-PC draws only 5 Watts, consuming in a day less power than a traditional PC consumes in 1 hour. You can leave fit-PC to work 24/7 without making a dent in your electric bill.

\subsection{Zonbu Computer:}

The Zonbu is a new, very energy efficient PC. The Zonbu consumes just one third of the power of a typical light bulb. The device runs the Linux operating system using a 1.2 gigahertz processor and 512 meg of RAM. It also contains no moving parts, and does even contain a fan. You can get one for as little as US\$99, but it does require you to sign up for a two-year subscription."

\subsection{Sunray thin client:}

Sun Microsystems is reporting increased customer interest in its Sun Ray, a thin desktop client, as electricity prices climb, according to Subodh Bapat, vice president and chief engineer in the Eco Responsibility office at Sun. Thin clients like the Sun Ray consume far less electricity than conventional desktops, he said. A Sun Ray on a desktop consumes 4 to 8 watts of power, because most of the heavy computation is performed by a server. Sun says Sunrays are particularly well suited for cost-sensitive environments such as call centers, education, healthcare, service providers, and finance. PCs have more powerful processors as well as hard drives, something thin clients don't have. Thus, traditional PCs invariably consume a substantially larger amount of power. In the United States, desktops need to consume 50 watts or less in idle mode to qualify for new stringent Energy Star certification.

\subsection{The Asus Eee PC and other ultra portables:}

The "ultra-portable" class of personal computers is characterized by a small size, fairly low power CPU, compact screen, low cost and innovations such as using flash memory for storage rat er than hard drives with spinning platters. These factors combine to enable them to run more efficiently and use less power than a standard form factor laptop. The Asus Eee PC is one example of an ultraportable. It is the size of a paperback, weighs less than a kilogram, has built-in Wi$\mathrm{Fi}$ and uses flash memory instead of a hard drive. It runs Linux too.

\subsection{Other implementations: \\ Notebooks}

Usually, notebooks are more modest than desktop PCs when it comes to the energy requirements. On average, notebook batteries last for less than two hours, so energy saving is an important issue for those who are away from a plug point for long durations. If you want to achieve maximum battery runtime then it's essential for a notebook to have energyefficient components. The warmer the external power supply unit, the higher the electricity consumption. Apple's MacBooks or Acer-models have intelligent charging electronics that ensure the current-flow sinks below 0.1 Watts after the battery is charged. Values less than 3.0 Watts, in Samsung's Q10, for instance, are acceptable. This is known as 'conservation charging.'

\section{Printers and multifunctional devices}

Usually, monochromatic laser printers require less electricity than color lasers. And this is true even in the standby mode. Color lasers use more energy when they go into the standby mode instead of the sleep mode. All color lasers require more than 10 Watts when they are in standby. To conserve energy, check the settings in the printer driver.

\section{Communications and network:}

W-LAN routers, DSL modems and DECT telephones do not have a standby mode since they must always be ready for operation. But low power consumption is a must since these devices are on 24 hours a day, seven days a week.

\section{External hard disks}

Users are increasingly buying 3.5 inch external hard disks as backup devices for desktop and notebook computers. These are also being used to extend the system storage. Once connected, it's easy to forget that its power supply continues to draw power, even when nothing is being read or written to the disk. Only a few models have sophisticated power-saving mechanisms; Seagate devices are quite commendable. Most devices do not have a 'Power' button. The 3.5-inch hard drives need 12 Volts and therefore they have an external power supply unit (power brick). But 2.5-inch drives require just 5 Volts and they can draw power from the PC via a USB cable. Since they draw power from the PC's power supply unit, the 2.5-inch drives will switch off automatically when the PC shuts down. Drive manufacturers are now incorporating features such as the reduced RPM low-power idle mode. 


\section{DVD and video}

Older DVD players and recorders are power hogs. Some devices consume up to 25 Watts in the standby mode and a switch-off button is absent. You can save energy in most such devices with a simple trick: The HF amplifier in DVD recorders isresponsible for consuming a good amount of electricity in the standby mode. The amplifier refreshes the incoming antenna signal for the television, which is perhaps connected with an antenna cable. If one places the recorder and the television next to each other and connects them to the antenna using a T-connector, this amplification is rendered useless. Many devices have the option of completely deactivating the HF output in the set-up. Older video recorders often have sliding switches for this.

\section{Cisco}

Some of the activities Cisco follows include: review of energy efficiency concepts, enhance and standardize recycling programs and green cleaning, explore transportation services and landscaping/parking for sustainability opportunities, incorporate LEED certification and energy collection data requests in future site selection criteria and standard lease agreements.

\begin{abstract}
Aladdin
Aladdin has a global initiative to 'Go Green.' From the earliest stages of product design, through manufacturing, use, and recycling, it ensures that its activities and products are environment-friendly. So its factories and production comply with ISO environmental standards. Aladdin claims that it is fully RoHS compliant too. It has set up recycling bins in all its offices for bottles, plastics, and paper. It encourages its employees to save paper too.[8]
\end{abstract}

\section{D-Link}

D-Link claims its 'green' products have been compliant with RoHS since 2006 and with WEEE since 2005. D-Link's Green Ethernet technology save power when desktop-toswitches are idle and optimized power usage on detection of cable length. Most switches today still consume considerable power even when a cable link or desktops-to switch is turned off. D-Link's Green Ethernet technology will put the port in a sleep mode, thus reducing power used by that port. Usually, most switches send enough power to sustain data over a $100 \mathrm{~m}$ cable regardless of the actual cable length. In a typical users' environment, however, the cable is usually less than $20 \mathrm{~m}$. But Green Ethernet technology will automatically detect the cable length and optimally adjust power usage to save energy.

\section{Climate Savers Overview}

It is started by Google and Intel to drive energy efficiency by increasing the energy efficiency of new PCs \& servers and promoting the use of power management We can reduce global CO2 emissions from the operation of computers by 54 million tons a year by 2010 . That's like taking 11 million cars off the road each year.

\section{CONCLUSION}

Businesses seeking a cost-effective way to responsibly recycle large amounts of computer equipment face a more complicated process. They also have the option of contacting the manufacturers and arranging recycling options. However, in cases where the computer equipment comes from a wide variety of manufacturers, it may be more efficient to hire a third-party contractor to handle the recycling arrangements. There exist companies that specialize in corporate computer disposal services both offer disposal and recycling services in compliance with local laws and regulations. Such companies frequently also offer secure data elimination services. So far, consumers haven't cared about ecological impact when buying computers, they've cared only about speed and price. But as Moore's Law marches on and computers commoditize, consumers will become pickier about being green. Devices use less and less power while renewable energy gets more and more portable and effective. New green materials are developed every year, and many toxic ones are already being replaced by them. The greenest computer will not miraculously fall from the sky one day, it'll be the product of years of improvements. The features of a green computer of tomorrow would be like: efficiency, manufacturing \& materials, recyclability, service model, self-powering, and other trends. Green computer will be one of the major contributions which will break down the 'digital divide', the electronic gulf that separates the information rich from the information poor.

\section{REFERENCES}

[1] Saurabh Kumar Garg and Rajkumar Buyya, "Green Cloud computing and Environmental Sustainability",site:

http://www.cloudbus.org/papers/Cloud-

EnvSustainability2011.pdf.

[2] A. Weiss. Computing in the Clouds. netWorker, 11(4):16-25, Dec. 2007.

[3] R. Buyya, C. S. Yeo, S. Venugopa. Market-oriented cloud computing: Vision, hype, and reality for delivering it services as computing utilities. In Proceedings of the 10th IEEE International Conference on High Performance Computing and Communications (HPCC-08, IEEE CS Press, Los Alamitos, CA, USA) 2008.

[4] Cisco. (2009). Cisco visual networking index:Forecast and methodology, 2009-2014. White paper. [Online]. Available:http://www.cisco.com.

[5] A. Weiss,"Computing in the clouds,[netWorker, vol. 11, no. 4, pp. 16-25, 2007.

[6] B. Hayes,"Cloud computing,Commun.ACM , vol. 51, no. 7, pp. 9-11, 2008.

[7] T. Singh and P. K. Vara,"Smart meteringthe clouds,inProc. IEEE Int. Workshops Enabling Technol., Infrastructures for Collaborative Enterprises, Groningen, The Netherlands, Jun.-Jul. 2009, pp. 66-71.

[8] D. Kondo, B. Javadi, P. Malecot, F. Cappello, and D. P. Anderson,Cost-benefit analysis of cloud 
computing versus desktop grids,in Proc. IEEE Int. Symp. Parallel Distrib. Process. Rome, Italy, May 2009, DOI: 10.1109/IPDPS.2009.5160911.

[9] R. Buyya, C. S. Yeo, and S. Venugopal,'Marketoriented cloud computing:Vision, hype, and reality for delivering IT services as computing utilities,in Proc. 10th IEEE Int. Conf. High Performance Comput. Commun., Dalian, China, Sep. 2008,pp. 513.

[10] A. Greenberg, P. Lahiri, D. A. Maltz, P. Patel, and S. Sengupta,"Towards a next generation data center architecture: Scalability and commoditization,in Proc. ACM Workshop Programmable Routers for Extensible Services of Tomorrow, New York, 2008, pp. 57-62.

[11 Open Cloud Manifesto. [Online]. Available:http://www.opencloudmanifesto.org/

[12] M. Armbrust, A. Fox, R. Griffith, A. D. Joseph,R. H. Katz, A. Konwinski, G. Lee, D. A. Patterson, A. Rabkin, I. Stoica, and M. Zaharia,"Above the clouds: A Berkeley view of cloud computing, Electr. Eng.Comput. Sci. Dept., Univ. California,Berkeley, CA, Tech. Rep. UCB/EECS-2009-28, Feb. 2009.
[13] L. M. Vaquero, L. Rodero-Merino,J. Caceres, and M. Lindner, "A break in the clouds: Towards a cloud definition,"SIGCOMM Comput. Commun. Rev., vol. 39,no. 1, pp. 50-55, 2009

[14] F. Seb'e, J. Domingo-Ferrer, A. Mart'ınez-Ballest'e, Y. Deswarte, and J.-J. Quisquater, "Efficient remote data possession checking incritical information .

[15] Cong Wang, Sherman S.M, Qian Wang, Kui Ren, Wenjing Lou "Privacy-Preserving Public Auditing for Secure Cloud Storage".

[16] http://en.wikipedia.org/wiki/Green computing

[17] 'Green IT For Dummies'-Hewlett Packard Limited Edition.

[18] Report of the Green Computing Task Group Green Computing and theEnvironment

[19] a b c San Murugesan, "Harnessing Green IT: Principles and Practices," IEEE IT Professional, January-February 2008, pp 24-33.

[20] "Green IT: Why Mid-Size Companies Are Investing Now"

[21] www.climatesaverscomputing.org

[22] Intelligent Computing Chip-Green Computing

[23] PC World Magazine, an article on Green Ethernet 